\title{
Functional correlation between olfaction and various sectioning of the lateral olfactory tract
}

Yasuyuki Sekiguchi $^{\mathrm{a}, \mathrm{b}}$, Nanae Fukushima ${ }^{\mathrm{a}, *}$, Kumiko Yokouchi $^{\mathrm{a}}$, Kyutaro Kawagishi ${ }^{\mathrm{a}}$, Shuichi Hirayama ${ }^{\mathrm{b}}$, Tetsuji Moriizumi ${ }^{\mathrm{a}}$

${ }^{a}$ Department of Anatomy, Shinshu University School of Medicine, 3-1-1 Asahi, Matsumoto, Nagano 390-8621, Japan

${ }^{b}$ Department of Neurosurgery, Shinshu University School of Medicine, 3-1-1 Asahi, Matsumoto, Nagano 390-8621, Japan

*Corresponding author. Tel.: +81-263-37-2593; fax: +81-263-37-3088.

E-mail address: nanae@shinshu-u.ac.jp (N. Fukushima).

The total number of pages: 16; figures: 8; tables: 0 


\section{ABSTRACT}

The lateral olfactory tract (LOT) is a central olfactory pathway, and efferent projections from the olfactory bulb are conveyed to the olfactory-related cortical structures via the LOT. The purpose of the present study is to determine the exact site of the LOT causing functional impairment in animals. After ablation of the right olfactory bulb, rats received rostrocaudal transection injuries on the left LOT at different levels between the olfactory bulb and the middle cerebral artery. Olfactory function of LOT-transected rats was studied by examining their olfactory ability to discriminate between the smell of water and cycloheximide solution, a strong repellent for rodents. Rats were divided into two groups based on their olfactory discriminative abilities. The olfaction positive $(+)$ group achieved $83 \% \pm 1 \%$ correct responses and the distances of the LOT-transected sites from the middle cerebral artery of this group ranged between 0.8 and $2.4 \mathrm{~mm}(n=$ 8). The olfaction negative (-) group achieved $48 \% \pm 1 \%$ correct responses and the distances of the LOT-transected sites from the middle cerebral artery ranged between 2.5 and $4.2 \mathrm{~mm}(n=10)$. From these data, we concluded that the site of the LOT critical for olfactory function is located approximately $2.5 \mathrm{~mm}$ from the middle cerebral artery.

Keywords:

Central olfactory tract

Transection injury

Olfaction

Neuronal tracer

Rat 


\section{Introduction}

The lateral olfactory tract (LOT) is a central olfactory pathway, and bulbar efferent projections are directed via the LOT to multiple ventrobasal cortical areas, collectively known as the olfactory cortices (Baker and Spencer, 1986; Haberly, 2001; Moriizumi et al., 1995; Ojima et al., 1984; Scott et al., 1980). The olfactory cortices include the anterior olfactory nucleus, olfactory tubercle, piriform cortex, and parts of the cortical amygdaloid nucleus and the entorhinal cortex. Olfactory perception is achieved through the neural circuits of the olfactory epithelium, olfactory bulb and olfactory cortices. Bulbar projections to the olfactory cortices are supposed to leave the LOT gradually to the adjoining olfactory cortices; rostrally to the anterior olfactory nucleus, medially to the olfactory tubercle, laterally to the piriform cortex, and caudally to parts of the cortical amygdaloid nucleus and the entorhinal cortex.

No data are available about the exact site of the LOT causing loss of olfaction. The aim of the present study is to determine the site of the LOT causing anosmia in animals. For this purpose, after direct visualization of the LOT, we made sharp transections on the LOT at different levels between the olfactory bulb and the middle cerebral artery. After removal of the olfactory bulb contralateral to the injured LOT, olfactory function was examined in the LOT-transected rats by their olfactory ability to discriminate between the smell of water and cycloheximide solution, a strong repellent for rodents (Fukushima et al., 2002; Moriizumi et al., 1994; Sakamoto et al., 2010). An anterograde neuronal tracing method was employed to confirm completeness of LOT transection and to exclude incomplete LOT-transected animals.

\section{Materials and methods}




\section{1. Animals}

Experiments were carried out in accordance with the National Institute of Health Guide for the Care and Use of Laboratory Animals and protocols were approved by our Institutional Animal Care and Use Committee. All efforts were made to minimize the number of animals used and their suffering. Female Wistar rats (12-16 weeks old, weighing 150-210 g) (Japan SLC Inc., Hamamatsu, Japan) were used in this study. Surgical manipulations were performed under general anesthesia by administering intraperitoneal injection consisting of a mixture of pentobarbital $(50 \mathrm{mg} / \mathrm{kg})$ and medetomidine $(10 \mathrm{mg} / \mathrm{kg})$. To reverse the anesthesia, antipamezole hydrochloride (2 $\mathrm{mg} / \mathrm{kg}$, i.p.) was injected.

\subsection{Identification of olfactory cortices by anterograde neuronal tracing}

Rats ( $n=3$ ) received multiple injections of an anterograde neuronal tracer, biotinylated dextran amine (BDA) (BDA-10000; Molecular Probes, Eugene, OR, USA), into the olfactory bulb (0.8-1.0 $\mu \mathrm{l})$. Three to five days after BDA injections, the rats were perfused through the heart with $4 \%$ paraformaldehyde under deep anesthesia with pentobarbital (80-100 mg/kg, i.p.). Brains were removed, post-fixed overnight in the same fixative, and soaked in 30\% sucrose for 2 days.

Olfactory bulbs were cut into sections $30-50 \mu \mathrm{m}$ thick in the horizontal or sagittal plane serially at $300-\mu \mathrm{m}$ intervals on a freezing microtome. Brains were cut into sections 30-50 $\mu \mathrm{m}$ thick in the coronal plane serially at 90 - or 300- $\mu \mathrm{m}$ intervals. One set of sections were processed for detection of BDA. After immersion in $0.3 \% \mathrm{H}_{2} \mathrm{O}_{2}$ for 30 min to suppress endogenous peroxidase activity, the sections were incubated overnight in $0.1 \mathrm{M}$ phosphate-buffered saline containing the avidin-biotin-peroxidase complex 
(1.5\%) and Triton X-100 (0.3\%). They were visualized with the Metal Enhanced DAB Substrate Kit (Thermo Fisher Scientific Inc., Pierce Biotechnology, Rockford, IL, USA). The sections were then mounted on coated slides and coverslipped with Entellan New media. Another set of sections were mounted on coated slides and stained with cresyl violet.

Fig. 1A-F show the LOT and its surrounding brain regions, all of which were strongly labeled with BDA transported from the olfactory bulb. The labeled regions included the anterior olfactory nucleus, olfactory tubercle, piriform cortex, cortical amygdaloid nucleus, and entorhinal cortex. The LOT and the olfactory cortices receiving afferent terminals from the olfactory bulb are summarized in Fig. 1G.

\subsection{Measurements of LOT-transected sites}

Rats were subjected to unilateral LOT transection on the left side. An approach was made from the ventrolateral aspect of the head between the eye and the ear. The LOT was clearly visualized as a white myelinated band ventral to the rhinal fissure. LOT transection was made between the olfactory bulb and the middle cerebral artery by inserting the tip of a knife (Ophthalmic Scleral MVR Knife, 25 gauge; Alcon, Tokyo, Japan). The brain was removed after transcardial perfusion with fixative. The location of the LOT-transected site was measured by the distance from the middle cerebral artery just overlying the LOT to the proximal edge of the transected LOT on the bulbar side with the aid of a micromeasure using a $0.1 \mathrm{~mm}$ scale under a binocular dissecting microscope (Fig. 2). The measurement was carried out at the midpoint in the mediolateral extent of the LOT, because the LOT had a broad fiber layer especially at the rostral part. 


\subsection{Experimental design}

Rats $(n=45)$ were subjected to unilateral ablation of the olfactory bulb on the right side and unilateral LOT transection on the left side. Various levels of LOT transections were made rostrocaudally between the olfactory bulb and the middle cerebral artery. After surgery, the rats were divided into several different cages $(26 \mathrm{~cm} \times 42 \mathrm{~cm} \times 18$ $\mathrm{cm})$ and each cage contained 3-4 rats. The animals were housed under standard laboratory conditions with a 12-h light/12-h dark cycle for 4 to 6 days. Room temperature was maintained at $22 \pm 1{ }^{\circ} \mathrm{C}$. Small pieces of fragmented solid food (standard rat chow) and water were supplied ad libitum. Forty percent (18/45) of the experimental rats died within $24 \mathrm{~h}$ after surgery.

After water deprivation for 3 days, the surviving LOT-transected rats $(n=27)$ were submitted to odor aversion behavior tests to examine their olfactory ability to discriminate $0.01 \%$ cycloheximide solution from water (Sakamoto et al., 2010). For control and behavioral comparison, the rats were subjected to unilateral $(n=7)$ or bilateral $(n=5)$ bulbectomy. Two bottles were offered to each rat, one containing $0.01 \%$ cycloheximide and the other containing water. Since the taste of cycloheximide solution is repulsive, rats remember the smell of the solution the first time they drink it and thereafter learn to avoid it by olfaction. When the rats drank water, the response was interpreted as a correct response, whereas when they drank cycloheximide solution, the response was interpreted as a wrong response. The number of correct responses was divided by the number of total trials for each rat and the percentages of correct responses were calculated in the experimental groups. At least 30 trials were performed for each rat and the percentages of correct responses were also calculated in the last 10 
trials. Data are provided as mean \pm standard error of the mean (SEM). Statistical significance of the means was evaluated by one-way analysis of variance followed by Dunnett test as post hoc analysis. $P$ values less than 0.05 were considered significant.

After the olfactory discrimination test, the LOT-transected rats received multiple injections of 0.8-1.0 $\mu \mathrm{l}$ of BDA into the remaining left olfactory bulb. As described previously, brain sections including the left olfactory bulb and the left olfactory cortices were examined for the presence of BDA transported in an anterograde fashion.

\section{Results}

\subsection{Exclusion of LOT-transected rats}

Two LOT-transected rats were excluded from the experiments because they were motionless and did not continue the olfactory discriminative test. In addition, rats $(\mathrm{n}=$ 4) in which the LOT was incompletely transected were excluded from experimental data collection. In such cases, BDA was transported from the olfactory bulb far caudally to the olfactory tubercle and the piriform cortex at a level near the optic chiasm. Finally, LOT transection procedures sometimes produced large cortical defects near the transected LOT. These rats $(n=3)$ were also excluded from experimental data collection.

\subsection{Correct responses of LOT-transected rats}

The LOT-transected rats were classified into two groups by their olfactory discriminative ability. The first group gave 1-4 wrong responses during the test with cyloheximide solution in the first set of 10 trials, thereafter they showed aversive behavior to cycloheximide solution. Their correct responses were greatly increased, as 
indicated by avoidance of cycloheximide and selection of water, in the second and third sets of 10 trials. After excluding the incomplete LOT-transected cases revealed by histological examination of the anterograde neuronal tracer (BDA), as described above, the percentage of correct responses of this group $(n=8)$ was $83 \% \pm 1 \%$ in all trials and $95 \% \pm 2 \%$ in the last 10 trials. These results were similar to the unilateral bulbectomy control group $(n=7)(88 \% \pm 2 \%$ in all trials and $97 \% \pm 2 \%$ in the last 10 trials) (Figs. 3 and 4).

The second group did not show aversive behavior to cycoheximide solution at any point during the test. The percentage of correct responses of this group ( $n=10)$ was $48 \% \pm 1 \%$ in all trials and $49 \% \pm 3 \%$ in the last 10 trials. These results were similar to the bilateral bulbectomy group $(n=5)(49 \% \pm 1 \%$ in all trials and $50 \% \pm 3 \%$ in the last 10 trials) (Figs. 3 and 4).

\subsection{Olfaction and LOT-transected sites}

Distances of the LOT-transected sites from the middle cerebral artery were $0.8,1.4$, 1.5, 1.6, 1.7, 1.9, 2.0 , and $2.4 \mathrm{~mm}$ in the rats with olfactory discriminative ability, while the distances were $2.5,2.6,2.7,3.1,3.2,3.3,3.5,3.8,3.9$, and $4.2 \mathrm{~mm}$ in the rats without olfactory discriminative ability. Figs. 5 and 6 show ventral aspects of the brains from the representative cases of the olfaction positive $(+)$ and olfaction negative (-) LOT-transected rats. Histological examinations revealed the injured LOT and its adjacent structures at different levels of the rostrocaudal axis (Fig. 7). Complete transections of the LOT were confirmed by the anterograde neuronal tracing in all cases, except for 4 cases with incomplete transections, as described previously. Fig. 8 shows representative cases of the olfaction positive $(+)$ and olfaction negative (-) 
LOT-transected rats, where BDA was traced from the injected olfactory bulb to the injured LOT, but not to the more caudal portions.

\section{Discussion}

The LOT is a unique and interesting brain structure because it can be clearly recognized macroscopically as a white band continuing from the olfactory bulb and can be directly approached from a ventrolateral direction in rats. Bulbar projections to the olfactory-related cortical structures, generally called the olfactory cortices, are conveyed via the LOT for very long distances. Prior to this study, there were no systematic studies on the relation between LOT lesions and olfactory functional loss, except for previous studies (Slotnick and Berman, 1979; Slotnick and Risser, 1990) reporting that LOT lesions did not substantially result in anosmia in experimental animals. By contrast, we found that LOT lesions did cause anosmia, if lesions were made on the proximal LOT near the olfactory bulb (Fukushima et al., 2002). However, there have been no data available to determine the exact site of the LOT required for olfactory function. For this purpose, we made sharp transections on the LOT along the rostrocaudal axis at different levels and examined olfactory function of the LOT-injured rats by measuring the distance of the transected site from the point of the middle cerebral artery traversing the LOT.

The following considerations are important in pursuing the present study. First, the unilateral olfactory bulb contralateral to LOT transections should be completely removed to examine the olfactory ability of the unilateral olfactory system on the LOT-transected side (Fukushima et al., 2002; Kawagishi et al., 2009; Moriizumi et al., 1994). This is because approximately $21 \%$ of the unlesioned, intact unilateral bulb has 
been reported to be sufficient for normal olfaction in bulb-ablation experiments ( $\mathrm{Lu}$ and Slotnick, 1998). As shown in Figs. 5 and 6, the olfactory bulbs were extensively ablated, often along with the caudal olfactory striae, and thus there were no cases of incomplete removal of the bulbs in our study. Second, transection injuries on the LOT should be restricted to the LOT without involving the surrounding olfactory cortices. However, combined cortical lesions, usually seen as large cortical defects particularly at the lateral part of the LOT, were inevitable in several cases (8\%: 3/39). These rats were excluded from further experimentation. Finally, the LOT should be completely transected, leaving behind no fiber connections to the olfactory cortices caudal to the transected LOT. The incomplete LOT-transected cases occurred mostly at the proximal LOT where small medial or lateral parts of the LOT tended to remain intact because of its wideness near the olfactory bulb. The percentage of such incomplete LOT transections in our study was $10 \%$ (4/39). Since the LOT gradually becomes narrow in width towards the middle cerebral artery, the incomplete LOT-transected cases occurred rarely at the more caudal LOT. Anterograde neuronal tracing employed in the present study was very useful for the confirmation of completeness of the LOT transection (Sakamoto et al., 2010).

In the olfaction positive (+) LOT-injured rats, the LOT-transected sites were located away from the olfactory bulb and near the middle cerebral artery. The brain regions between the olfactory bulb and the LOT-transected sites included the anterior olfactory nucleus, the rostral region of the olfactory tubercle, and the rostral region of the piriform cortex. The distances of the LOT-transected sites from the middle cerebral artery ranged between 0.8 and $2.4 \mathrm{~mm}$. In the olfaction negative (-) LOT-injured rats, the LOT-transected sites were located close to the olfactory bulb and away from the middle cerebral artery. The brain regions between the olfactory bulb and the LOT-transected 
sites included only the anterior olfactory nucleus with or without the anterior most region of the piriform cortex. The distances of the LOT-transected sites from the middle cerebral artery ranged between 2.5 and $4.2 \mathrm{~mm}$. From these data, we concluded that the exact site of the LOT critical for olfactory function is located about $2.5 \mathrm{~mm}$ from the middle cerebral artery, although the respective specific site should be determined by each odor. It is noteworthy that the rostral end of the olfactory tubercle is located about $2.5 \mathrm{~mm}$ from the middle cerebral artery as shown in Fig. 2, thus serving as a clear landmark in terms of functional significance. Since the distance between the bulbar caudal end and the middle cerebral artery is approximately $5.0 \mathrm{~mm}$, the rostral end of the olfactory tubercle is situated midway between the bulb and the artery.

It is wonder whether the functional impairment depends on the transection of olfactory pathway or the destruction of olfactory cortices by knife cut. However, the possibility that the impairment produced by the direct destruction was minor because the injured sites were considerably restricted compared with the whole olfactory cortex as shown in Figs. 5-8. Minimal cortical areas essential for olfactory functional preservation seem to be too small compared to the very large sizes of the olfactory cortices as shown in Fig. 1G. In this connection, Lu and Slotnick (1998) examined olfactory function in rats with varying degrees of bulbectomy and reported that bulbectomized rats with bulbar saving scores of more than $21 \%$ had no deficits in odor detection or discrimination tasks. Similarly, we also reported that the minimal number of unilateral bulbar projection neurons required for normal olfaction is about $20 \%$ of the normal value in rats with varying degrees of injuries to the LOT (Fukushima et al., 2002). Thus, the relatively small cortical sizes speculated from our LOT-injured experimental data might be enough for functional preservation. 


\section{Acknowledgments}

This work was supported by a Grant-in-Aid for Scientific Research from the Ministry of Education, Science, Sports, and Culture of Japan. 


\section{References}

Baker, H., Spencer, R.F., 1986. Transneuronal transport of peroxidase-conjugated wheat germ agglutinin (WGA-HRP) from the olfactory epithelium to the brain of the adult rat. Exp. Brain Res. 63, 461-473.

Fukushima, N., Oikawa, S., Yokouchi, K., Kawagishi, K., Moriizumi, T., 2002. The minimum number of neurons in the central olfactory pathway in relation to its function: a retrograde fiber tracing study. Chem. Senses 27, 1-6.

Haberly, L.B., 2001. Parallel-distributed processing in olfactory cortex: new insights from morphological and physiological analysis of neuronal circuitry. Chem. Senses 26, 551-576.

Kawagishi, K., Yokouchi, K., Fukushima, N., Sakamoto, M., Sumitomo, N., Moriizumi, T., 2009. Determination of functionally essential neuronal population of the olfactory epithelium for nipple search and subsequent suckling behavior in newborn rats. Brain Res. 1276, 50-57.

Lu, X.-C.M., Slotnick, B.M., 1998. Olfaction in rats with extensive lesions of the olfactory bulbs: implications for odor coding. Neuroscience 84, 849-866.

Moriizumi, T., Sakashita, H., Furukawa, M., Kawano, J., Okoyama, S., Kitao, Y., Kudo, M., 1995. Electron microscopic study of synaptogenesis and myelination of the olfactory centers in developing rats. Exp. Brain Res. 103, 385-392.

Moriizumi, T., Tsukatani, T., Sakashita, H., Miwa, T., 1994. Olfactory disturbance induced by deafferentation of serotonergic fibers in the olfactory bulb. Neuroscience 61, 733-738.

Ojima, H., Mori, K., Kishi, K., 1984. The trajectory of mitral cell axons in the rabbit olfactory cortex revealed by intracellular HRP injection. J. Comp. Neurol. 230, 
Sakamoto, M., Yokouchi, K., Sekiguchi, Y., Fukushima, N., Kawagishi, K., Kakegawa, A., Sumitomo, N., Moriizumi, T., 2010. Re-evaluation of spontaneous regeneration of the lateral olfactory tract. Neurosci. Res. 68, 15-21.

Scott, J.W., McBride, R.L., Schneider, S.P., 1980. The organization of projections from the olfactory bulb to the periform cortex and olfactory tubercle in the rat. J. Comp. Neurol. 194, 519-534.

Slotnick, B.M., Berman, E.J., 1979. Transection of the lateral olfactory tract does not produce anosmia. Brain Res. Bull. 5, 141-145.

Slotnick, B.M., Risser, J.M., 1990. Odor memory and odor learning in rats with lesions of the lateral olfactory tract and mediodorsal thalamic nucleus. Brain Res. 529, 23-29. 


\section{Legend for figures}

Fig. 1. (A) Ventral view of a normal rat brain. An arrow indicates the position of the middle cerebral artery. (B) An injection site of BDA into the left olfactory bulb. (C-F) BDA was transported to the olfactory cortices. C-F correspond to c-f in (A). (G) Olfactory cortices receiving BDA (+) bulbar terminals. Asterisks indicate the cortical amygdaloid nucleus $(* * *)$ and entorhinal cortex $(* *)$. AON: anterior olfactory nucleus; LOT: lateral olfactory tract; OB: olfactory bulb; OT: olfactory tubercle; PC: piriform cortex. Scale bar: $1 \mathrm{~mm}$.

Fig. 2. The distance from the middle cerebral artery to the transected LOT between the arrows was measured to be $2.5 \mathrm{~mm}$.

Fig. 3. Correct responses of the four groups of the experimental animals as expressed by percentages in all trials. (A) Control rats with unilateral bulbectomy (88\%). (B) LOT-transected rats with olfaction (83\%). (C) LOT-transected rats without olfaction (48\%). (D) Rats with bilateral bulbectomy (49\%). No significant differences are found between unilaterally bulbectomized (A) and olfaction positive (+) LOT-transected (B) rats, and between olfaction negative (-) LOT-transected (C) and bilaterally bulberctomized (D) rats. ${ }^{* *} P<0.01$ vs. control.

Fig. 4. Correct responses of the four groups of the experimental animals as expressed by percentages in the last 10 trials. (A) Control rats with unilateral bulbectomy (97\%). (B) LOT-transected rats with olfaction (95\%). (C) LOT-transected rats without olfaction (49\%). (D) Rats with bilateral bulbectomy (50\%). ** $P<0.01$ vs. control.

Fig. 5. LOT-transected sites of olfaction positive $(+)$ rats. The distances from the middle cerebral artery to the transected LOT between the arrows were 0.8 (A), 1.6 (B) and 2.4 (C) mm, respectively. 
Fig. 6. LOT-transected sites of olfaction negative (-) rats. The distances from the middle cerebral artery to the transected LOT between the arrows were 3.2 (A), 2.7 (B) and 2.5 (C) mm, respectively.

Fig. 7. Microscopic views of LOT-transected sites. (A, B) Olfaction negative (-) rats. (C, D) Olfaction positive $(+)$ rats. Arrows indicate the injured sites of the LOT. AON: anterior olfactory nucleus; OT: olfactory tubercle; PC: piriform cortex. Cresyl violet stain. Scale bar: $1 \mathrm{~mm}$.

Fig. 8. Rats with the LOTs transected at 3.2 (A-E, olfaction-negative), 2.5 (F-J, olfaction-negative) and 1.5 (K-O, olfaction-positive) $\mathrm{mm}$ from the middle cerebral artery. Arrows indicate the LOT-injured sites and arrowheads point to BDA reaction product. Note the absence of BDA (+) bulbar terminals in the olfactory cortices caudal to the transected LOTs. Scale bar: $1 \mathrm{~mm}$. 


\section{Fig 1}

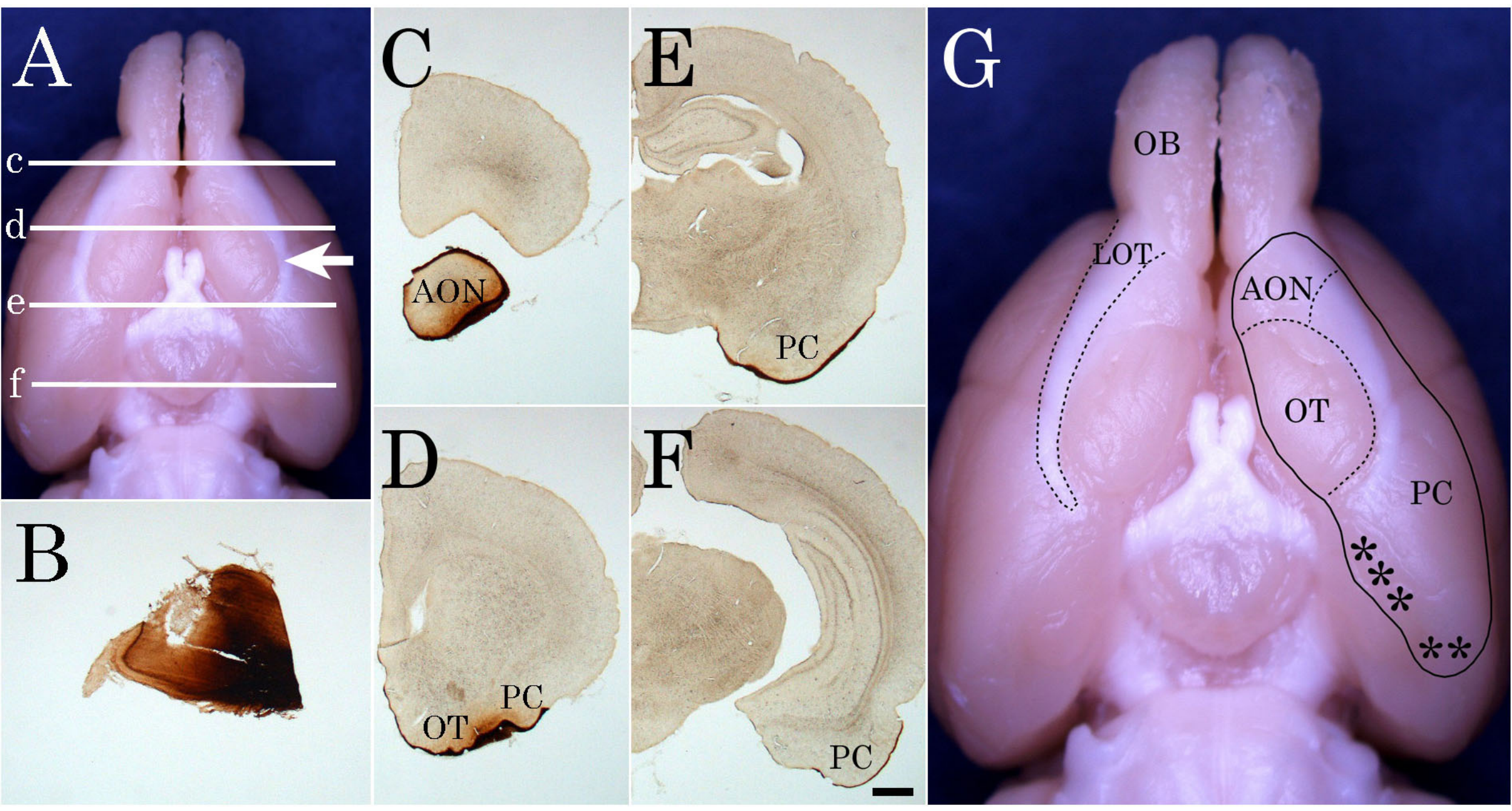


Fig 2

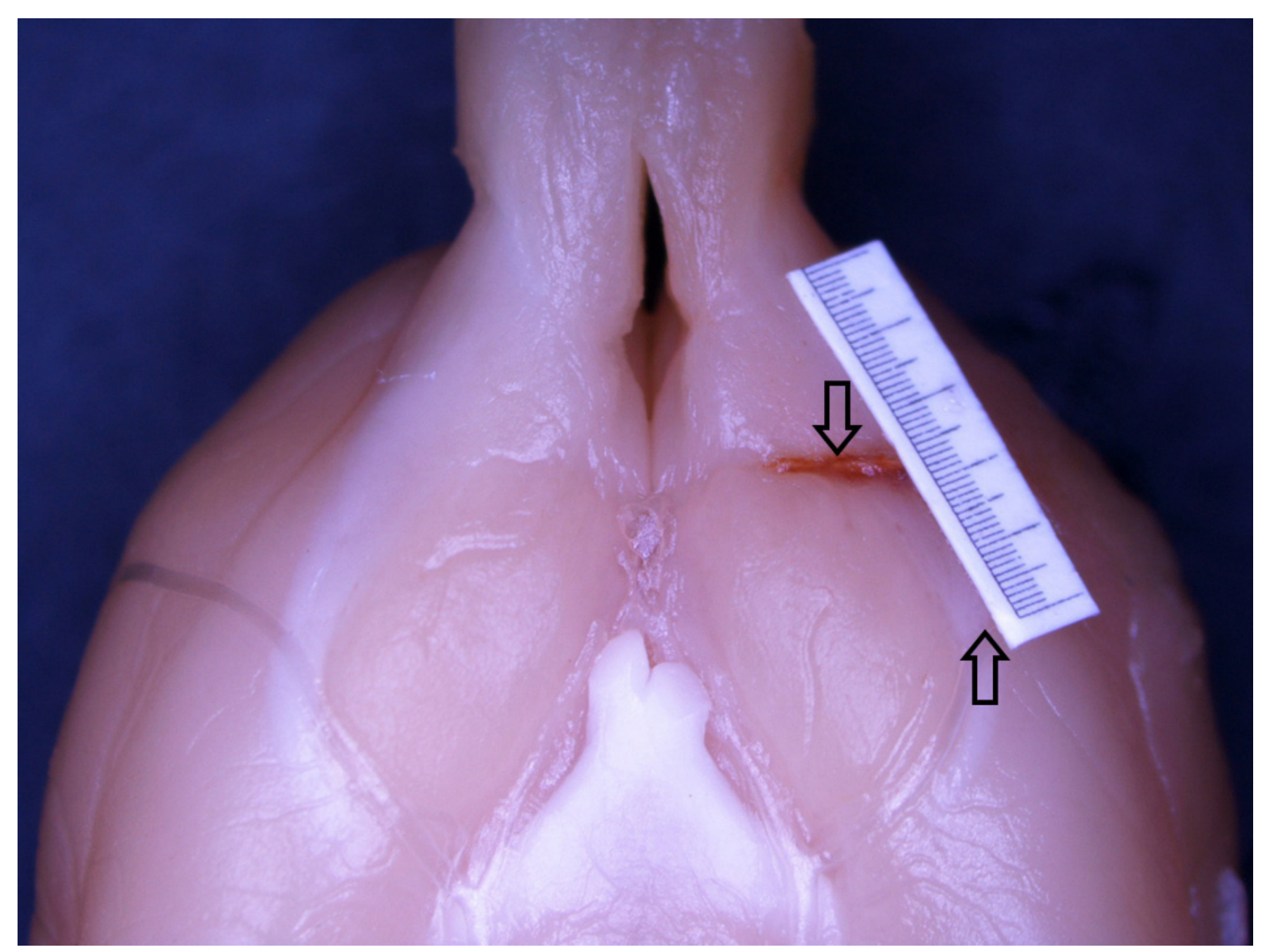




\section{Fig 3}

\section{Fig 4}

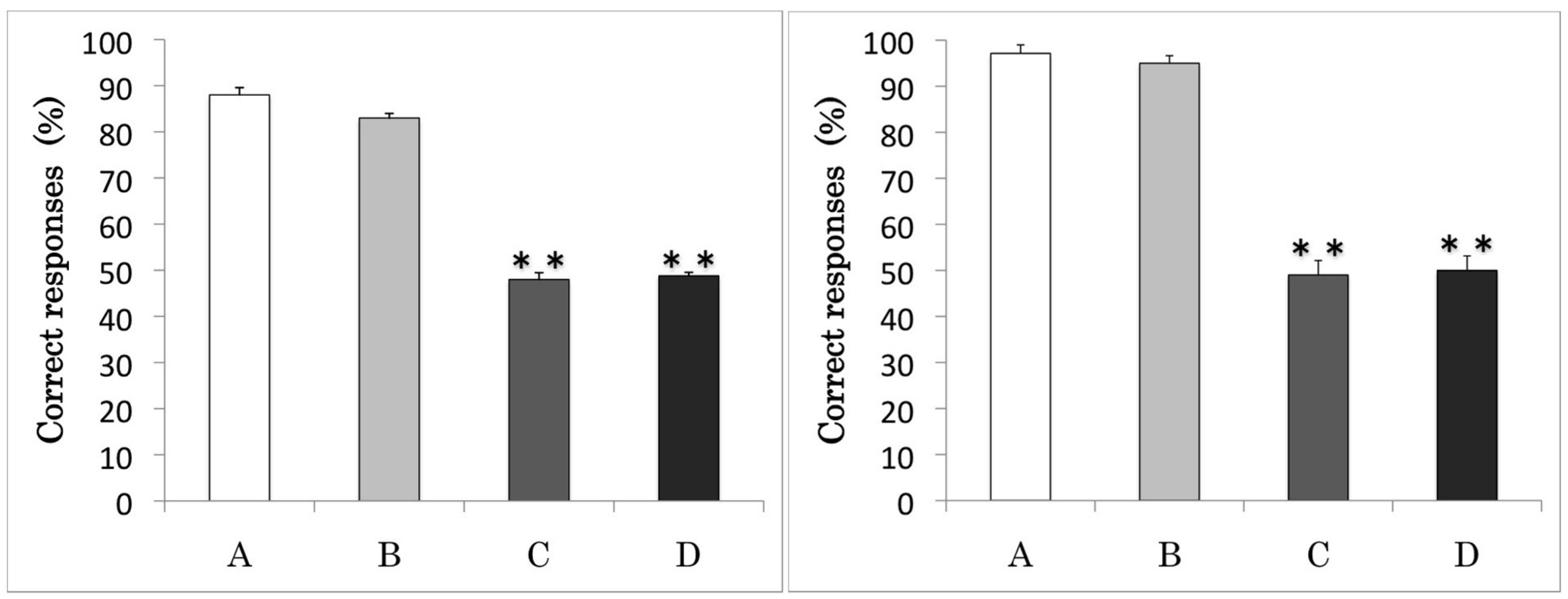




\section{Fig 7}

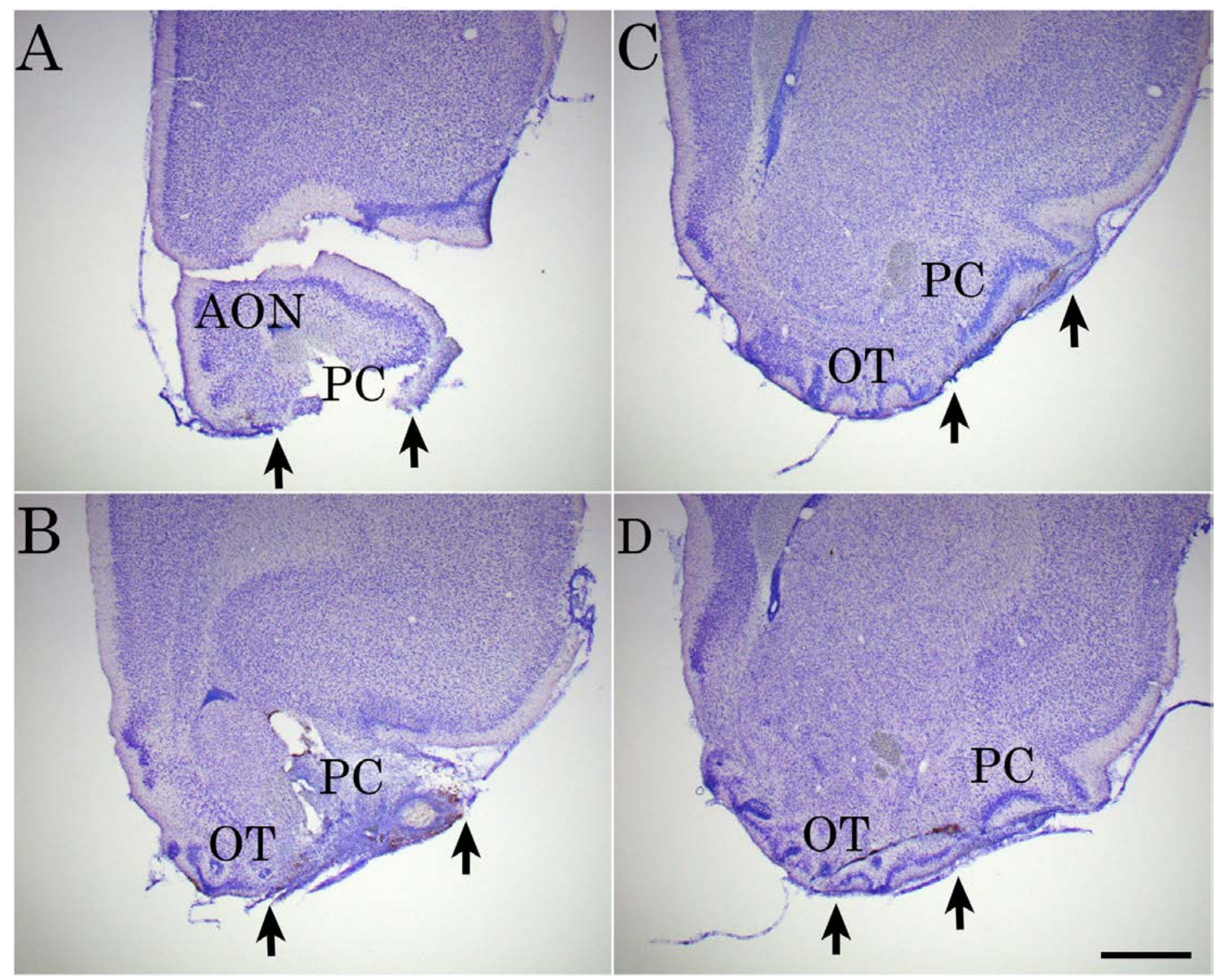


$A \quad 4 \quad F \quad-4$

Fig 8

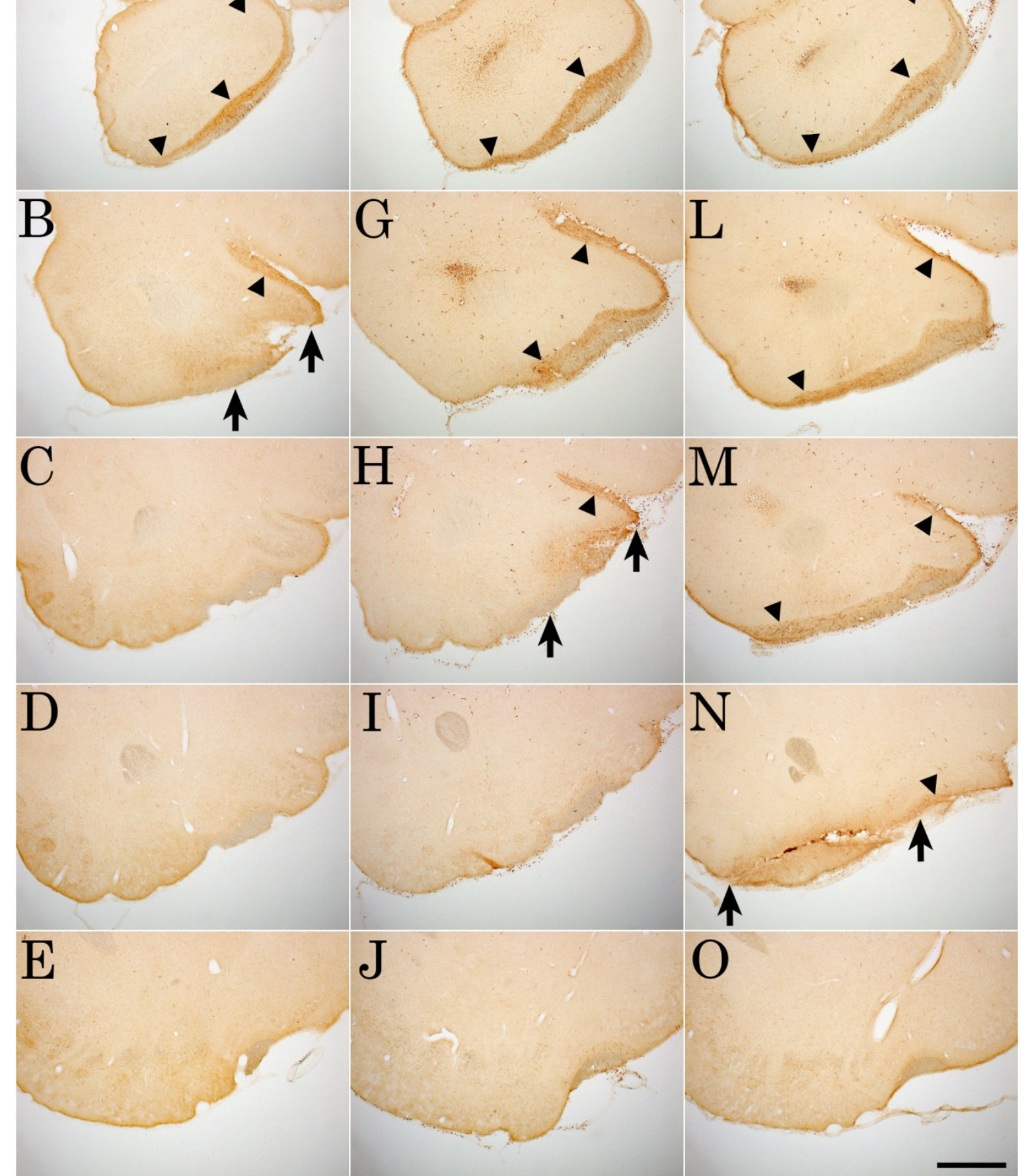

